\title{
EL TIPO DE INCENTIVO COMO DETERMINANTE EN EL ATRACTIVO DE LA PROMOCIÓN DE VENTA EN TURISMO RURAL. EFECTO MODERADOR DEL SEXO, LA EDAD Y LA EXPERIENCIA
}

\section{TYPE OF INCENTIVE AS A DETERMINANT OF THE APPEAL OF SALES PROMOTION IN RURAL TOURISM. THE MODERATING EFFECT OF GENDER, AGE AND EXPERIENCE}

\author{
Salvador Del Barrio García, Lorenza López Moreno y Dolores M. Frías Jamilena ${ }^{1}$ \\ UNIVERSIDAD DE GRANADA
}

\section{RESUMEN}

El turismo rural es un sector de gran importancia dentro del turismo español y requiere de la puesta en práctica de instrumentos promocionales que permitan conseguir una mayor atracción de clientes. En este trabajo se pretende investigar el atractivo de distintos tipos de incentivos promocionales (promociones congruentes y no congruentes tanto monetarias como no monetarias), así como el efecto interacción entre la propensión a las promociones, variables demográficas y el efecto experiencia de los individuos con el turismo rural. Los resultados del trabajo ponen de manifiesto una influencia significativa del tipo de promoción sobre su atractivo. Concretamente se ha demostrado que las promociones de ventas congruentes (excursiones y cuidado del medio ambiente) con la actividad de turismo rural son más atractivas que las no congruentes no monetarias (regalos y sorteos). Sin embargo, este mayor atractivo de las herramientas congruentes no se manifiesta en uno de los tipos de promociones no congruentes monetarias (descuentos en precio). También, se ha puesto de manifiesto que la edad y el sexo moderan parcialmente el efecto de la propensión sobre el atractivo promocional y que la experiencia no modera dicho efecto.

Palabras clave: Propensión a la promoción, atractivo promocional, turismo rural, experiencia con el turismo rural.

\footnotetext{
${ }^{1}$ Departamento de Comercialización e Investigación de Mercados. Facultad de Ciencias Económicas y Empresariales. Universidad de Granada. Campus Cartuja, s/n, 18071 Granada. dbarrio@ugr.es; 1lopezm@ugr.es; dfrias@ugr.es.
} 


\section{ABSTRACT}

Rural tourism is a sector of major importance for Spanish tourism and as such requires promotional tools to be employed that enable greater numbers of clients to be attracted. The present work seeks to investigate the appeal of different types of promotional incentives (congruent and non-congruent - the latter covering monetary and non-monetary promotions). The work also examines the effect of interaction between promotion proneness, demographic variables and the effect of individuals' prior experience of rural tourism. The findings reveal that the type of promotion has a significant influence on its appeal. It is demonstrated that, in rural tourism, congruent sales promotions (excursions and contributions made to the care of the environment) are more attractive than non-congruent, non-monetary promotions (gifts and prize draws). However, this greater appeal of congruent promotional tools is not to be found in one type of non-congruent monetary promotions (namely price discounts). It is also shown that age and gender partially moderate the effect of promotion proneness on promotional appeal, and that prior experience does not moderate this effect.

Keywords: Promotion proneness, promotional appeal, rural tourism, prior experience of rural tourism.

\section{INTRODUCCIÓN}

Una de las alternativas de turismo que ofrece España es el turismo rural que ha experimentado la mayor tasa de crecimiento de forma ininterrumpida desde sus inicios hasta la actualidad (Instituto NaCional DE Estadística, INE, 2010). De hecho, en la última década, entre los años 2001 y 2009 (ambos inclusive) el crecimiento mostrado por la oferta se situó en el $152,63 \%$ para el número de alojamientos rurales, y para la demanda en el 123,68\% para el número de turistas rurales (INE, 2010). Este sector representa el $9,02 \%$ de los establecimientos turísticos y el $6,07 \%$ de la oferta de plazas turísticas (INE, 2010).

La verdadera importancia del sector turístico rural no se centra solamente en los volúmenes de oferta y demanda alcanzados, sino que se encuentra en su capacidad para contribuir al desarrollo sostenible de las economías y, de forma concreta, al desarrollo sostenible del medio rural (FLEISCHER Y FELSENSTEIN, 2000; BARKE, 2004; ORganización Mundial DEL Turismo, OMT, 2007; EUROPEAN COMISSION, 2010). En la actualidad el turismo rural constituye una forma alternativa de hacer turismo, con entidad propia (BlANCO, 1996; GARCÍA, 1996; Consejería de Turismo, Comercio Y Deportes de la Junta de Andalucía, 2004), donde la principal motivación del turista se centra en el contacto con el medio rural y/o con sus recursos (FUENTES, 1995:76; VALDÉS, 1996).

Si bien, en los últimos años el turismo rural se ha visto favorecido por un mayor dinamismo de la demanda, en la actualidad su desarrollo se enfrenta a retos importantes. En primer lugar, la alta intensidad competitiva, con una oferta turística cada vez mayor en cantidad y variedad de alternativas turísticas que hacen difícil para los diferentes destinos turísticos mantener y defender sus cuotas de mercado (OKUMUS Y Hemmington, 1998; CAmisón, 2000 y Ávila Y BARRADO, 2005). En segundo lugar, el mayor grado de exigencia del turista como consecuencia de una mayor experiencia y conocimiento (CAMisÓn, 2000).

Como resultado a esta situación de mayor nivel de exigencia por parte de la demanda y de mayor intensidad competitiva por parte de la oferta, las empresas de turismo rural requieren la puesta en práctica de instrumentos promocionales que permitan conseguir una mayor atracción de clientes, especialmente en etapas de crisis como la actual en las que existe un preocupante descenso de la demanda turística. De hecho en los últimos años el sector turístico 
se ha caracterizado por una alta intensidad promocional. Son numerosos los estudios que ponen de manifiesto que las promociones de ventas tienen una clara influencia en el comportamiento final de compra del consumidor (Álvarez y VÁzQuez, 2005; 2008). No obstante, no todos los consumidores reaccionan de la misma forma a las promociones, ya que su efectividad dependerá de aspectos como la propensión del consumidor a la promoción o el tipo de promoción a la que se encuentre expuesto, entre otros.

Sin embargo, son pocos los trabajos que analizan la efectividad de las promociones de ventas en el ámbito del turismo en general y, menos aún, en el caso del turismo rural.

En este trabajo se pretende abordar un doble objetivo. Primero, conocer la influencia del tipo de promoción sobre el atractivo de las promociones que se realizan en el sector de turismo rural, diferenciando entre promociones congruentes y no congruentes con el tipo de turismo objeto de estudio, y en estas últimas, entre promociones monetarias y no monetarias. Segundo, estudiar si la influencia de la propensión a la promoción en el atractivo promocional de diferentes tipos de herramientas promocionales está moderada por variables sociodemográficas como la edad y el sexo y por otras variables como la experiencia del turista con el turismo rural.

\section{REVISIÓN DE LA LITERATURA}

\subsection{Determinantes de la efectividad de la promoción de ventas}

La efectividad de la promoción de ventas depende de una serie de factores como la propensión del consumidor a la promoción (HENDERSON, 1987; BLATTBERG Y NESLIN, 1990; SCHNEIDER Y CURRIM, 1991; WAKEFIELD Y BARnes, 1996; Teunter, Wierenga y KloeK, 1999; DelVecchio, 2005), el tipo de promoción (Shimp y Kavas, 1984; BaWA y Shoema-
KeR, 1989; BlattBerg y Neslin, 1990; MitTal, 1994; Mela, Gupta y Lehman, 1997; Mela, Jedidi y Bowman, 1998; KRISHNA Y ZHANG, 1999; PALAZÓN, 2005), el tipo de beneficio que aporta e incluso la congruencia que tenga ese beneficio con el producto o servicio que se esté promocionando (Dowling Y UnCles, 1997; Chandon, WANSINK Y LAURENT, 2000; KWOK Y UNCLES, 2002; RoEHM, Pullins Y Roehm JR, 2002).

La propensión a la promoción fue definida por Lichtenstein, Netemeyer Y BURToN (1990) como "la propensión general a responder ante una promoción debido a que aparece como una oferta". Es decir, se trata de la tendencia a utilizar la información que proporcionan las promociones para la toma de decisiones de compra, de manera que los consumidores más propensos serán quienes modifiquen su comportamiento de compra para beneficiarse del incentivo temporal que incluyen (WAKEFIELD Y BARNES, 1996).

No obstante, no todos los consumidores presentan el mismo interés por los incentivos promocionales que desarrollan las empresas, sino que existen grupos de consumidores que muestran una predisposición positiva hacia la información que la promoción les transmite que posteriormente será utilizada para llevar a cabo sus decisiones de compra (PAlazón y DelgaDO, 2009).

La literatura recoge investigaciones que han analizado la propensión a las promociones considerando distintas perspectivas. Así, hay trabajos que han estudiado la propensión a las promociones en función de la herramienta promocional utilizada (cupones, rebajas, folletos, etc.) (SCHNEIDER y CURRIM, 1991; LiCHTENSTEIN, NeTEMEYER y BuRTON, 1995, LiCHTENStein, Burton y NeteMEyer, 1997b; Ailawadi, Neslin y Gedenk, 2001, Swaminathan Y BAWA, 2005).

Otros, como por ejemplo, ScHNEIDER Y CURRIM (1991) estudian la propensión a la promoción atendiendo al comportamiento del individuo, diferenciando entre propensión a la pro- 
moción activa y pasiva. Mediante la propensión activa, es más probable que se produzca una búsqueda intensiva para localizar las promociones interesantes, refiriéndose en este caso a la sensibilidad del consumidor hacia los folletos y cupones distribuidos fuera del establecimiento. Por otro lado, la propensión pasiva hace referencia a la sensibilidad del consumidor hacia los folletos y cupones que encuentra en el establecimiento, limitándose la búsqueda de promociones a los puntos de venta. En esta línea, AILAWADI et al. (2001) distinguen entre promociones exteriores e interiores. Las promociones exteriores tienen lugar fuera del punto de venta (folletos y cupones distribuidos por establecimientos o fabricantes) y requieren un esfuerzo importante por parte del consumidor, de ahí que se relacionen con la propensión activa de SCHNEIDER y CURRIM (1991). Por su parte, las promociones interiores se caracterizan por realizarse en el punto de venta, el consumidor no las conoce hasta que hace la compra, requieren un escaso esfuerzo, estando más próximas a la propensión pasiva de SCHNEIDER Y CURRIM (1991). También MARTínez Y Montaner (2004; 2007) diferencian entre propensión a las promociones interiores y exteriores.

En general, la literatura previa ha hallado que los consumidores más propensos a las promociones harán una mejor evaluación de éstas (PALAZÓn, 2005) y reaccionarán de manera positiva ante cualquier tipo de acción promocional (Shimp y KaVAs, 1984; Price, Feick y Guskey-Federouch, 1988; Alford y Biswas, 2002; D`Astous y JACOB, 2002; CHANDRASHEKARAN Y GREWAL, 2003; VICDAN Y SUN, 2008).

Otro de los factores que influyen en la efectividad de la promoción de ventas es el tipo de promoción utilizado. Las promociones pueden clasificarse según múltiples criterios. Para CHANDON et al. (2000) uno de los de mayor relevancia es aquel relacionado con la naturaleza de la misma: monetaria y no monetaria. Las primeras engloban herramientas promocionales que ofrecen beneficios de ahorro y calidad, relacionados éstos con una reducción en el pre- cio normal del producto o con un incremento de la cantidad ofrecida al mismo precio. Es el caso de los descuentos, cupones, precios paquete o incrementos de la cantidad, que representan distintas formas de modificación de la relación precio-cantidad para así incrementar el valor percibido de la oferta (PeATtiE Y Peattie, 1996). Sin embargo, las promociones no monetarias ofrecen unos beneficios de diversión/entretenimiento, exploración y de expresión de valor que no implican la modificación del precio sino el ofrecimiento de algo extra con el producto como por ejemplo premios, regalos, concursos, sorteos o muestras gratuitas.

En la literatura se ha tratado ampliamente los efectos que diferentes tipos de promociones ejercen en la respuesta del consumidor. Por ejemplo, BAWA Y SHOEMAKER (1989) se centraron en la reacción de los consumidores ante el uso de cupones, mientras que KRISHNA Y ZHANG (1999) analizaron la influencia de la duración de los cupones en su efectividad. También se ha estudiado la influencia del conocimiento que tiene el consumidor sobre los precios para el caso de las promociones basadas en las reducciones de precios (KRISHNA, CURRIM Y SHOEMAKER, 1991). El esfuerzo que debe realizar el consumidor para beneficiarse de la promoción resulta también de especial interés, para lo que se ha concluido que las promociones que no requieren ningún esfuerzo por parte del consumidor, como las reducciones inmediatas en el precio, están recomendadas en consumidores sensibles al precio (WALTERS, 1991; SIVAKUMAR Y RAJ, 1997).

La promoción en precio es una de las más utilizadas por la literatura. LICHTENSTEIN et al. (1995), trataron de diferenciar las promociones de ventas atendiendo a su influencia en el precio. De este modo, es posible hablar de promociones en precios que tendrán como resultado una reducción del precio de venta, y de promociones no centradas en el precio que no producirán tal reducción (LICHTENSTEIN et al., 1995). Es razonable suponer que los consumidores 
más sensibles a los precios también mostrarán una mayor sensibilidad ante las promociones centradas en los mismos (Lichtenstein, NETEMEYER Y BURTON, 1997a).

En la industria turística se utilizan de forma mayoritaria promociones de carácter monetario o basadas en reducciones de precios, aunque existe acuerdo en afirmar que las promociones no monetarias pueden añadir valor al consumidor y permitir alcanzar los objetivos de comunicación y promoción (Peattie y Peattie, 1996). En consonancia con esta apreciación, estos autores analizan el potencial de los sorteos como una de las técnicas de promoción de ventas no monetaria más popular. En esta línea de promociones no monetarias, MONTY Y SKIDMORE (2003) analizan la predisposición a pagar por un conjunto de servicios adicionales en el sector de la hostelería y el alojamiento y que podría considerarse también como una herramienta de promoción de ventas no basada en el precio.

No obstante, en la literatura se pone de manifiesto que además del tipo de promoción, la efectividad de la promoción de ventas depende de la congruencia que tenga el beneficio que aporte al consumidor la promoción con el producto o servicio que se esté promocionando (ChANDOn et al., 2000; MARTínez; MonTANER Y PINA, 2007). De hecho si la información que recibe un individuo es congruente será recordada mejor y la actitud y, por tanto, su eficacia será significativamente mayor en comparación con la información incongruente o irrelevante (CANTOR Y MisCHEL, 1979; TAYLOR Y CROCKER, 1981; LYNCH Y SCHUlER, 1994; DOWLING y UNCLES, 1997; CHANDON et al., 2000; KWOK y UNCLES, 2002; Roenm et al., 2002).

La congruencia e incongruencia son importantes porque influyen en el tipo de procesamiento que los consumidores eligen para realizar sus evaluaciones y formar actitudes, tal y como pone de manifiesto la teoría de la categorización. Esta teoría indica que las personas que se enfrentan con un nuevo objeto a evaluar tratarán de hacerlo coincidir con una categoría preexistente. Si encuentran una coincidencia, tenderán a utilizar un proceso de transferencia de actitud para evaluar el nuevo objeto en el cual las evaluaciones hacia la categoría se transfieren al mismo. Este modo implica un mínimo esfuerzo de procesamiento mucho más simplista y holístico (WANG, BEATTY Y MOTHERSBAUGH, 2009). D'Astous y LANDREVILLE (2003) analizan la percepción que tienen los clientes de las promociones de ventas basadas en premios e incluyen como aspecto importante a tener en cuenta la adecuación entre el premio y la categoría de producto que se está promocionando. Concluyen que el atractivo del premio es determinante para que la oferta sea bien acogida, y que en el caso de no resultar muy atractivo su valoración también será positiva si el premio tiene un alto grado de ajuste con el bien promocionado. Señalan que la promoción de ventas que incluya un premio que se ajuste bien a la categoría de producto se percibirá como menos manipuladora.

En la literatura existen trabajos que manifiestan una clara necesidad de seguir investigando en la eficacia de las promociones. Por ejemplo, LUCAS y BowEN (2002) muestran la necesidad de analizar la efectividad de las promociones de ventas en el sector del ocio y YoON et al. (2010) señalan la importancia de analizar el modo en el que el consumidor percibe diferentes niveles de descuentos en precio así como diferentes formatos de descuentos dentro del contexto de los servicios.

Además, en el sector turístico en general, se tiende a analizar de forma conjunta un amplio número de herramientas promocionales, o a asumir que un tipo de herramienta promocional representa a las demás. Por este motivo, PEATTIE y Peattie (1996) sugieren la importancia y la necesidad de analizarlas de forma individualizada y atendiendo al sector industrial en el que se aplican.

En este trabajo se pretende avanzar un poco más en la literatura comprobando de forma individualizada el efecto sobre el atractivo de distintos tipos de promociones (monetarias y no monetarias) y diferenciando entre las que 
son congruentes o no congruentes con el servicio. Así, si los incentivos que se le ofrecen al turista son congruentes con la actividad de turismo desarrollada, el consumidor los procesará dentro de la categoría de turismo rural y usará un proceso de transferencia de actitudes para evaluarlos. Como indica la literatura, esa congruencia generará en el turista una evaluación y actitud positiva hacia ese tipo de incentivos. Dentro de las promociones no congruentes cabe distinguir a su vez entre promociones de carácter monetario y no monetario. De acuerdo a los párrafos anteriores se plantean las siguientes hipótesis:

H1: El atractivo de las promociones de ventas congruentes con la actividad de turismo rural será significativamente mayor que el de las promociones de ventas no congruentes monetarias.

H2: El atractivo de las promociones de ventas congruentes con la actividad de turismo rural será significativamente mayor que el de las promociones de ventas no congruentes no monetarias.

\subsection{El efecto moderador del sexo, la edad y la experiencia del turista en la relación entre la propensión a la promoción y el atractivo promocional}

En el proceso de decisión de compra del consumidor se realiza un balance entre los beneficios que puede obtener de una promoción con los costes que debe soportar para beneficiarse de ella. Interesa señalar que ni los costes ni los beneficios son valorados de igual forma por todos los consumidores, de tal manera que dependiendo de cuál sea su perfil psicográfico y socioeconómico responderán de manera diferente a los distintos estímulos promocionales.
AIlAWADI et al. (2001) identifican un conjunto de rasgos sociodemográficos y psicográficos que influyen en la importancia que tienen los distintos beneficios para cada consumidor, debiéndose considerar, además, los costes asociados a la compra del producto promocionado (Tabla 1). Llegar a conocer cuál es el perfil del consumidor propenso a las promociones puede ayudar de manera muy positiva en el diseño del plan promocional (BAWA Y SHOEMAKER, 1989; LAROCHE et al., 2003).

De forma tradicional se ha considerado que los productos en promoción eran adquiridos por los consumidores con las rentas más bajas y por los más sensibles al precio. Sin embargo, estudios recientes manifiestan que la compra de productos en promoción se realiza no sólo por motivos económicos sino también porque al consumidor le entretiene, le genera un sentimiento de comprador inteligente, mejora su imagen ante los demás y sacia su deseo de explorar (CHANDON et al., 2000; AILAWADI et al., 2001; VILlalBA, 2005). Por tanto, inicialmente los trabajos sobre la influencia de las herramientas promocionales se centraban en identificar variables sociodemográficas, a las que se unieron variables relacionadas con los hábitos de compra (SCHNEIDER Y CURRIM, 1991), el tipo de visita que lleva a cabo el cliente al establecimiento (PARREÑO; LEEFLANG Y VAN DOORN (2009), llegándose a describir incluso perfiles psicográficos de los consumidores propensos a las promociones (LICHTENSTEIN et al., 1990, AILAWADI et al., 2001; MARTÍNEZ Y MONTANER, 2004; 2007).

MARTÍNEZ Y MONTANER $(2004 ; 2007)$ concluyen que, en términos generales, las personas preocupadas por los precios son propensas a las promociones, pero que éste no es el único aspecto a considerar, sino que características como la impulsividad, la innovación y el disfrute al realizar la compra también influyen en algunas de las propensiones que identifican. 
TABLA 1

\section{Rasgos psicográficos y sociodemográficos asociados a los beneficios y costes de la compra de productos en promoción}

\begin{tabular}{|c|c|c|c|}
\hline & & Características psicográficas & Características sociodemográficos \\
\hline \multirow{2}{*}{$\begin{array}{l}\text { Beneficios } \\
\text { utilitarios }\end{array}$} & Ahorros & $\begin{array}{l}\text { Preocupados por los precios } \\
\text { Restricciones económicas }\end{array}$ & Nivel de ingresos \\
\hline & Calidad del producto & Preocupados por la calidad & \\
\hline \multirow{3}{*}{$\begin{array}{l}\text { Beneficios } \\
\text { hedonistas }\end{array}$} & Entretenimiento & Disfrutar comprando & Edad \\
\hline & Exploración & $\begin{array}{l}\text { Innovación } \\
\text { Búsqueda de variedad } \\
\text { Impulsividad }\end{array}$ & \\
\hline & Auto-expresión & $\begin{array}{l}\text { Experto de mercado } \\
\text { Búsqueda de reconocimiento }\end{array}$ & Sexo \\
\hline \multirow{4}{*}{ Costes } & Costes de cambio & $\begin{array}{l}\text { Lealtad a la marca } \\
\text { Lealtad al establecimiento }\end{array}$ & \\
\hline & Costes de búsqueda & $\begin{array}{l}\text { Planificación } \\
\text { Presión de tiempo }\end{array}$ & $\begin{array}{l}\text { Hijos } \\
\text { Situación laboral }\end{array}$ \\
\hline & Costes de pensar & Necesidad de comprensión & Educación \\
\hline & Costes de inventario & Espacio percibido para almacenar & Tipo de residencia \\
\hline
\end{tabular}

Fuente: adaptado de AILAWADI et al. (2001, p. 74).

En la literatura se encuentra un amplio número de trabajos en los que se analiza la influencia de variables sociodemográficas en la efectividad de las herramientas promocionales (PeChTl, 2004; KWOK y UnCles, 2005; CARPENTER Y MOORE, 2007; KWON Y KWON, 2007). Variables como la educación (KWON Y KwON, 2007), los ingresos (AILAWADI et al., 2001; KWON Y KwON, 2007), la edad (LiCHTENSTEIN et al., 1997a; CARPENTER y MOORE, 2007) y el sexo (PeChtl, 2004; Kwon y Kwon, 2007; CARPENTER y MoOre, 2007). Otra variable que parece moderar la efectividad de las herramientas promocionales es la experiencia con el tipo de viaje (RAGHUBIR y CORFMAN, 1999).

En el ámbito turístico, la literatura también ha mostrado que variables demográficas como la edad, el sexo, los ingresos, el nivel educativo y el número y composición del grupo de viaje influyen en el proceso de búsqueda de información y en la decisión de compra (VAN RAAIJ Y FRANCKEN, 1984; FODNESS Y MURRAY, 1997; THORNTON, SHAW Y WiLliams, 1997; ZALATAN, 1998). Como ejemplo es de destacar el estudio de Duman y Mattila (2003), en el que se aborda la relación entre un conjunto de variables demográficas y aspectos relacionados con el tipo de viaje en el comportamiento de los turistas de cruceros que reciben algún tipo de descuento (por viajes especiales, por el número de integrantes en el grupo, por la antelación en la reserva, por ofertas especiales para niños, cupones, etc.). Como variables demográficas proponen la edad, el sexo, el nivel educativo y el nivel de ingresos; y respecto a las características del viaje utilizan el número de personas 
que viajan juntas, la composición del grupo, el tamaño del crucero, la duración del viaje, el momento en el que se realizó y la experiencia en este tipo de viajes. Concluyen que la edad, la experiencia previa con estos viajes y el sexo, tienen una influencia significativa en la utilización de descuentos como herramienta de promoción. En concreto, hallaron que a medida que aumenta la edad de los encuestados se reduce la probabilidad de recibir descuentos. Respecto a la experiencia, señalan que los viajeros más experimentados obtendrán más ventajas de los descuentos puesto que estarán más familiarizados con la búsqueda de mejores ofertas. Finalmente, concluyen que las mujeres manifiestan una mayor tendencia a utilizar los descuentos que los hombres, mostrando una actitud más activa en la búsqueda de ofertas especiales para la planificación de estos viajes. Estas conclusiones son muy similares a las obtenidas en otros sectores (LICHTENSTEIN et al., 1997a; MAZUNDAR y PAPATLA, 1995; CARPENTER Y MOORE, 2007; KWON Y KWON, 2007).

No obstante, no en todos los trabajos se obtienen los mismos resultados. El estudio de KWOK Y UNCLES (2005), que resulta interesante por contradictorio, analiza el efecto de diferentes variables culturales en las promociones sin encontrar que este efecto sea significativo, al tiempo que contrastan la teoría de la congruencia inicialmente estudiada por CHANDON et al. (2000). Obtienen que la dirección de los efectos entre el producto y el tipo de promoción es la opuesta a la prevista.

A pesar de que en este sector se utiliza de forma intensiva la promoción de ventas (PEATTIE y PeAtTIE, 1996), la investigación existente al respecto resulta muy reducida, más aún cuando se trata de analizar de forma conjunta la propensión a las promociones, las variables sociodemográficas o el grado de repetición de los viajes en la evaluación de la efectividad de las diferentes herramientas promocionales. De ahí la importancia de continuar analizando estas variables sociodemográficas, así como el tipo de herramienta promocional utilizada y su congruencia con el producto promocionado, al objeto de tratar de aclarar cuál es su influencia real.

Con este objetivo se propone estudiar el efecto moderador del sexo, la edad y la experiencia en la relación entre la propensión a las promociones y el atractivo de las diferentes herramientas promocionales (congruentes y no congruentes) propuestas inicialmente, planteándose las siguientes hipótesis:

H3: El efecto de la propensión a la promoción sobre el atractivo promocional de las promociones está moderado por el sexo, siendo mayor para las mujeres que para los hombres.

H3a: El efecto de la propensión a la promoción sobre el atractivo promocional de las promociones congruentes está moderado por el sexo, siendo mayor para las mujeres que para los hombres.

H3b: El efecto de la propensión a la promoción sobre el atractivo promocional de las promociones no congruentes monetarias está moderado por el sexo, siendo mayor para las mujeres que para los hombres.

H3c: El efecto de la propensión a la promoción sobre el atractivo promocional de las promociones no congruentes no monetarias está moderado por el sexo, siendo mayor para las mujeres que para los hombres.

H4: El efecto de la propensión a la promoción sobre el atractivo promocional de las promociones está moderado por la edad, siendo mayor para los turistas más jóvenes.

H4a: El efecto de la propensión a la promoción sobre el atractivo promocional de las promociones congruentes está moderado por la edad, siendo 
mayor para los turistas más jóvenes.

H4b: El efecto de la propensión a la promoción sobre el atractivo promocional de las promociones no congruentes monetarias está moderado por la edad, siendo mayor para los turistas más jóvenes.

H4c: El efecto de la propensión a la promoción sobre el atractivo promocional de las promociones no congruentes no monetarias está moderado por la edad, siendo mayor para los turistas más jóvenes.

H5: El efecto de la propensión a la promoción sobre el atractivo promocional de las promociones está moderado por la experiencia, siendo mayor para los turistas con más experiencia.

H5a: El efecto de la propensión a la promoción sobre el atractivo promocional de las promociones congruentes está moderado por la experiencia, siendo mayor para los turistas con más experiencia.

H5b: El efecto de la propensión a la promoción sobre el atractivo promocional de las promociones no congruentes monetarias está moderado por la experiencia, siendo mayor para los turistas con más experiencia.

H5c: El efecto de la propensión a la promoción sobre el atractivo promocional de las promociones no congruentes no monetarias está moderado por la experiencia, siendo mayor para los turistas con más experiencia.

\section{METODOLOGÍA}

Para cumplir con los objetivos propuestos en la investigación se diseñó una metodología basada en la recogida de información mediante cuestionarios cerrados y estructurados a una muestra de turistas que habían realizado al menos una estancia en alojamientos turísticos rurales de Andalucía. La razón para elegir este ámbito geográfico para nuestro estudio es doble. Por una parte, Andalucía es el destino preferido para los viajes de los residentes en España $(18,1 \%)$ (Instituto DE Estudios TuRísticos, IET, 2008). Por otra parte, Andalucía se ha constituido en los últimos años como uno de los destinos preferidos a nivel nacional para realizar turismo rural. En el año 2008, Andalucía ocupaba el cuarto lugar por número de plazas disponibles en establecimientos rurales en el total de las regiones españolas, con el 8,18\% del total (INE, 2011).

Dado que para poder realizar el estudio empírico y poder contrastar las hipótesis planteadas se necesitaba una muestra de turistas que hubieran tenido al menos una experiencia de turismo rural en el último año, se contó con la colaboración de la Asociación de Hoteles Rurales de Andalucía (AHRA) que incluye entre sus asociados a 62 establecimientos rurales repartidos por toda la geografía andaluza, que representan en torno al $37 \%$ del total de plazas de turismo rural disponibles en Andalucía. Para ello, se contactó con miembros de la Dirección de AHRA a los que se les explicó el objetivo de la investigación y se les propuso su colaboración a cambio de recibir un informe final de gestión. La Dirección contactó con los hoteles asociados explicando el proyecto propuesto por los investigadores y animando a colaborar con ellos. Concretamente, las instrucciones que la Dirección mandó a sus hoteles asociados fue la siguiente: En el momento en el que los clientes lleguen al hotel, al hacer el check-in, se les debe informar que investigadores de la Universidad de Granada en colaboración con AHRA están llevando a cabo un estudio sobre la satisfacción de los huéspedes de establecimientos rurales de Andalucía. Para lo cual, es importante solicitarles su colaboración para que accedan a responder a una 
encuesta telefónica pasadas unas cuantas semanas desde su estancia en el hotel, para lo que se les debe pedir su teléfono de contacto. Se les debe recordar que para agradecer su colaboración todos los huéspedes que concluyan la encuesta participarán en el sorteo de 3 estancias de dos noches en régimen de alojamiento y desayuno en uno de los hoteles de la asociación que ellos elijan".

Finalmente, participaron 23 de los 62 hoteles asociados $(37,10 \%)$, repartidos entre las 8 provincias que componen la Comunidad Autónoma de Andalucía, lo cual supone una muestra representativa de los hoteles rurales de AHRA, cuya oferta hotelera como se ha comentado con anterioridad a su vez representa cerca de 4 de cada 10 plazas de turismo rural en Andalucía. Un total de 243 turistas decidieron participar en el estudio y proporcionaron su teléfono de contacto y su autorización para llamarles y hacerles el cuestionario. Pasadas cuatro semanas se contactó con todos ellos (Computer Assisted Telephone Interview-CATI) y finalmente se obtuvieron 164 respuestas válidas, lo que representa un $67,49 \%$ del total. La tabla 2 muestra la ficha técnica del estudio.

TABLA 2

Ficha técnica

\begin{tabular}{|l|l|}
\hline Universo & Clientes de alojamientos de turismo rural \\
\hline Ámbito geográfico & Andalucía \\
\hline Método de recogida de información & Entrevista telefónica asistida por ordenador (CATI) \\
\hline $\begin{array}{l}\text { Tamaño de la muestra y tasa de } \\
\text { respuesta }\end{array}$ & $\begin{array}{l}164(67,49 \% \text { de un total de } 243 \text { clientes que aceptaron participar en el } \\
\text { estudio })\end{array}$ \\
\hline Error muestral $\left(^{*}\right)$ & $7,65 \%$ \\
\hline Nivel de confianza (*) & $95 \%, \mathrm{Z}=1,96, \mathrm{p}=\mathrm{q}=0,5$ \\
\hline Procedimiento de muestreo & $\begin{array}{l}\text { Discrecional (por conveniencia): clientes que han visitado un alojamiento } \\
\text { rural }\end{array}$ \\
\hline Trabajo de campo & Diciembre 2007 - enero 2008 \\
\hline
\end{tabular}

(*) En el supuesto de que se cumplieran los requisitos del muestreo aleatorio.

En cuanto a los instrumentos de medida utilizados, la variable dependiente a analizar es el atractivo promocional, una medida bastante precisa de la evaluación global de un individuo hacia un determinado incentivo promocional (ChANDON et al., 2000). En esta misma línea, la literatura sobre comunicación de marketing ha considerado el nivel de atractivo o agrado de un instrumento de comunicación como uno de los mejores indicadores de la eficacia del mismo (HALEy y BALDinger, 1991), constituyéndose en un importante mediador en la formación de actitudes hacia la marca (MACKENZIE, LUTZ Y BELCH, 1986).

Teniendo en cuenta los objetivos de la investigación propuestos, el procedimiento seguido fue plantear seis diferentes tipos de acciones promocionales y analizar su grado de atractivo entre la muestra de turistas rurales. Como promociones no congruentes monetarias se eligieron los "descuentos en precios sobre el importe final en factura" y la "entrega de vales o talones con noches 
extra para próximas estancias", por ser dos de los tipos de incentivos promocionales que más se utilizan en el sector turístico, en general, y en el rural, en particular. Dentro de las promociones no congruentes no monetarias con la categoría de producto se seleccionaron la "entrega de regalos tales como bolsa de viaje o recuerdos de la zona" y "la participación de concursos y sorteos para próximas estancias en algunos de los hoteles pertenecientes a la Asociación AHRA", incentivos que aunque no son tan frecuentes como los anteriores se emplean en algunas ocasiones en el sector turístico. La selección de los incentivos promocionales con una alta congruencia con la actividad de turismo rural y con beneficios hedónicos se realizó teniendo en cuenta la experiencia pasada de los establecimientos rurales pertenecientes a AHRA así como la opinión al respecto de algunos directivos de la Asociación. Así, finalmente se establecieron incentivos consistentes en la "oferta desde los establecimientos rurales de excursiones y actividades al aire libre de manera gratuita" y "la cesión por parte del alojamiento rural de un porcentaje del precio pagado por el turista al cuidado del entorno natural tal como repoblación forestal de la zona y protección de animales en peligro de extinción".

Todos los individuos debían valorar el grado de atractivo que les suponía cada uno de esos seis tipos de incentivos promocionales planteados mediante una escala de diferencial semántico de 5 puntos (1: nada atractivo, 5: muy atractivo). Concretamente, la cuestión que se les planteaba a los individuos muestrales era la siguiente: "Señale el grado de atractivo que tiene cada una de las siguientes promociones en el supuesto de que ahora mismo tuviera que decidirse por una oferta de alojamiento rural (suponga que todos los incentivos tienen el mismo valor económico)".

Las variables independientes empleadas fueron la propensión general a las promociones, la experiencia con el destino turístico y el sexo y la edad de los turistas. La propensión a las promociones se midió con una escala tipo Likert de 3 ítems y 5 puntos (1: totalmente en desacuerdo, 5: totalmente de acuerdo): "Tengo marcas favoritas pero la mayor parte de las veces compro la marca que está en promoción", "Comparado con mis familiares y amigos, es más probable que compre la marca que está en promoción", "Prefiero esperar para poder aprovecharme de una ventaja promocional". Esta escala fue adaptada de la original propuesta por LiCHTENSTEIN, RidWAY Y NETEMEYER (1993) que ha sido ampliamente referida y empleada en la literatura, y adaptada y utilizada al ámbito español por otros autores como GÁZQUEZ Abad y De CANNIÈRe (2010); PAlazón (2005) o Palazón y Delgado (2009).

La medida de la experiencia con el destino de turismo rural se realizó a través del número de días que el turista había estado en Andalucía en el último año alojándose en establecimientos de turismo rural. Esta escala se ha adaptado del trabajo de Duman y MatTILA (2003) utilizada para valorar la experiencia de los turistas en cruceros. Finalmente, la edad del encuestado se midió a través de 4 categorías: 18-29 años, 30-44 años, 45-65 años, más de 65 años.

\section{ANÁLISIS DE DATOS}

\subsection{Descripción de la muestra}

En el perfil sociodemográfico que presenta la muestra final obtenida existe un gran equilibrio en cuanto al sexo de los encuestados con un $50,61 \%$ de hombres y un $49,39 \%$ de mujeres. Respecto a la distribución de la muestra en términos de edad el $25 \%$ corresponde a turistas entre 18 y 29 años, el 52\% entre 30 y 44 años, el $18 \%$ entre 45 y 65 años y sólo el 5\% a turistas de más de 65 años ${ }^{2}$. Los clientes entrevista-

\footnotetext{
${ }^{2}$ Para el análisis de datos posterior se agruparon las dos últimas categorías de edad en una sola de más de 44 años que incluía el 23\% de la muestra.
} 
dos tienen en su mayoría un nivel de estudios superiores o al menos secundarios, lo que representa más del $80 \%$ de la muestra. Con respecto al lugar de procedencia de los turistas, el $84,76 \%$ de la muestra proceden de la propia comunidad autónoma de Andalucía frente al $15,24 \%$ restante que lo hacen desde fuera de Andalucía, principalmente de Madrid y el levante mediterráneo.

\subsection{Análisis de validez y fiabilidad de la escala de propensión a la promoción}

$\mathrm{Al}$ objeto de poder incorporar en el análisis la variable propensión a la promoción medida mediante una escala multi-ítem y poder construir una puntuación suma de la misma que permita su tratamiento como variable independiente en el análisis posterior de regresión, se procedió a evaluar previamente su fiabilidad y validez mediante análisis factorial confirmatorio (AFC). En primer lugar, se analizó la distribución muestral de los datos comprobándose que éstos no seguían una distribución multinormal. Este hecho unido al reducido tamaño muestral disponible nos llevó a escoger como método de estimación más adecuado el de Máxima Verosimilitud Robusto tal y como recomiendan SATORRA Y BENTLER (1986). El AFC realizado mediante el software Lisrel 8.8 mostró que la escala poseía buenas propiedades psicométricas, ya que presentaba validez convergente y buenos niveles de fiabilidad. Además, los índices de bondad de ajuste presentan valores muy adecuados (véase tabla 3 ).

Una vez comprobada la validez y consistencia interna de esta escala se procedió a calcular la escala suma con un rango entre 3 (mínima propensión de un turista a las promociones) y 15 (máxima propensión de un turista a las promociones).

TABLA 3

\section{Evaluación de la escala de medida propensión hacia la promoción}

\begin{tabular}{|c|c|c|}
\hline Item & Coeficientes estandarizados $\lambda$ & Fiabilidad Individual $\left(\mathbf{R}^{2}\right)$ \\
\hline PROP1 & $0,88^{*}$ & 0,78 \\
\hline PROP2 & $0,84(9,11)$ & 0,70 \\
\hline PROP3 & $0,67(8,30)$ & 0,45 \\
\hline Fiabilidad Compuesta & & 0,8422 \\
Varianza Extraída & 0,6432 \\
de Cronbach & 0,8037 \\
RMSEA & 0,0000 \\
p-value & 0,9846 \\
\hline
\end{tabular}

* Parámetro fijado a 1 .

\subsection{Contraste de hipótesis}

Las hipótesis $\mathrm{H} 1$ y $\mathrm{H} 2$ proponían que el atractivo promocional de promociones de ventas congruentes con la actividad de turismo rural será significativamente mayor que el atractivo de promociones que incluyan incentivos no congruentes, ya sean monetarios o no monetarios, respectivamente. La tabla 4 muestra las puntuaciones medias de atractivo para cada uno de los seis tipos de incentivos promocionales considerados. Los dos incentivos 
promocionales que son valorados como más atractivos por la muestra de turistas rurales son los descuentos directos en precio y la cesión por parte del alojamiento rural de un porcentaje del precio pagado para el cuidado del entorno natural con valores ligeramente superiores a 4. Le siguen la oferta de excursiones y actividades al aire libre, los vales con noches extra y las promociones no monetarias tales como entrega de regalos y participación en sorteos y concursos. Al objeto de contrastar las anteriores hipótesis se llevaron a cabo diferentes test de diferencias de medias que mostraron claramente que las promociones congruentes con las actividades de turismo rural se perciben significativamente más atractivas que aquellas otras no congruentes y no monetarias tales como regalos y sorteos $(\mathrm{p} \leq 0,05)$, confirmándose por tanto $\mathrm{H} 2$. Los resultados, en cambio, no fueron tan claros para $\mathrm{H} 1$, ya que el contraste entre las promociones basadas en excursiones y las basadas en vales de descuento resultó cuasi-significativo $(\mathrm{p} \leq 0,10) \mathrm{y}$, especialmente, el contraste entre las promociones basadas en incentivos de actividades de cuidado del medioambiente y las basadas en descuentos en precios no fue significativo $(\mathrm{p}>0,10)$. En cambio, los contrastes entre las promociones basadas en excursiones y las basadas en descuentos y entre las basadas en el cuidado del medioambiente y las basadas en vales de descuento sí que resultaron significativos $(\mathrm{p} \leq 0,05)$. Estos resultados nos llevan a confirmar parcialmente $\mathrm{H} 1$.

TABLA 4

Estadísticas básicas del atractivo promocional

\begin{tabular}{|c|c|c|}
\hline Tipos de promociones & Media & Desv. Típ. \\
\hline \multicolumn{3}{|l|}{ Promociones no congruentes monetarias } \\
\hline Descuentos en precio (Atdescuentos) & 4,10 & 0,98 \\
\hline Vales noches extra (Atvales) & 3,50 & 1,27 \\
\hline \multicolumn{3}{|l|}{ Promociones no congruentes no monetarias } \\
\hline Entrega de regalos (Atregalos) & 3,00 & 1,38 \\
\hline Participación en concursos y sorteos (Atsorteos) & 2,91 & 1,34 \\
\hline \multicolumn{3}{|l|}{ Promociones congruentes con el turismo rural } \\
\hline Oferta de excursiones y actividades al aire libre gratuitas (Atexcursiones) & 3,75 & 1,23 \\
\hline Cesión de \% importe del alojamiento para el cuidado del entorno natural (Atcuidado) & 4,01 & 1,14 \\
\hline $\begin{array}{l}\text { Contrastes Promociones Congruentes vs Promociones no Congruentes Monetarias } \\
\text { Atexcursiones vs. Atdescuentos (t-value: }-2,8839, \mathrm{p} \text {-value: } 0,0042 \text { ) } \\
\text { Atexcursiones vs. Atvales (t-value: } 1,8104, \mathrm{p} \text {-value: } 0,0711) \\
\text { Atcuidado vs. Atdescuentos (t-value: }-0,8335, \mathrm{p} \text {-value: } 0,4052 \text { ) } \\
\text { Atcuidado vs. Atvales (t-value: } 3,8007, \mathrm{p} \text {-value: } 0,0001 \text { ) }\end{array}$ & & \\
\hline $\begin{array}{l}\text { Contrastes Promociones Congruentes vs Promociones no Congruentes no Monetarias } \\
\text { Atexcursiones vs. Atregalos (t-value: } 5,1969, \mathrm{p} \text {-value: } 0,0000) \\
\text { Atexcursiones vs. Atsorteos (t-value: } 5,8908, \mathrm{p} \text {-value: } 0,0000) \\
\text { Atcuidado vs. Atregalos (t-value: } 7,2067, \mathrm{p} \text {-value: } 0,0000) \\
\text { Atcuidado vs. Atsorteos (t-value: } 7,9663, \mathrm{p} \text {-value: } 0,0000)\end{array}$ & & \\
\hline
\end{tabular}


Por su parte, para el contraste de las hipótesis $\mathrm{H} 3, \mathrm{H} 4$ y H5, dadas las métricas de las variables objeto de análisis, se planteó un modelo de regresión lineal para cada una de las seis promociones establecidas (dos congruentes, dos no congruentes monetarias y dos no congruentes no monetarias). La variable dependiente fue el grado de atractivo del incentivo promocional considerado y las variables independientes la propensión general hacia la promoción y las interacciones de esta variable con el sexo, la edad y la experiencia con el destino.

Dado que el sexo y la edad son variables cualitativas (nominal y categórica, respectivamente) para su introducción en el modelo de regresión fue necesario transformarlas en variables dummy o indicadoras. Así, para la variable sexo se creó una variable dummy (Dmujer) codifica- da como 1 cuando el sexo del encuestado era mujer y como 0 cuando era hombre. Para el caso de la edad dado que se tenían 3 categorías (18-29; 30-44; más de 44) se crearon 2 variables dummy: D30-44 codificada como 0 cuando el turista tenía de 18 a 29 años o más de 44 años y como 1 cuando su edad se situaba entre 30 y 44 años; y Dmas44 codificada como 0 cuando la edad se situaba por debajo de los 44 años y 1 cuando la edad era superior a los 44 . Esta forma de proceder, creando $n-1$ variables dummy cuando una variable categórica tiene $n$ categorías se realiza para evitar la trampa de la variable categórica que daría lugar a una situación de multicolinealidad perfecta en caso de crear $n$ variables dummy (JUDGE, et al., 1988).

Realizadas estas transformaciones se plantearon seis modelos de regresión con la siguiente especificación:

\section{- Promociones congruentes}

atexcursiones $=\alpha+\beta_{1}$ prop $+\beta_{2}$ prop $*$ Dmujer $+\beta_{3}$ prop $*$ D30 $-44+\beta_{4}$ prop $*$ Dmas $44+\beta_{5}$ prop $* \exp +\varepsilon$ atcuidado $=\alpha+\beta_{1}$ prop $+\beta_{2}$ prop $*$ Dmujer $+\beta_{3}$ prop $*$ D30 $-44+\beta_{4}$ prop $*$ Dmas $44+\beta_{5}$ prop $* \exp +\varepsilon$

- Promociones no congruentes monetarias

atdescuentos $=\alpha+\beta_{1}$ prop $+\beta_{2}$ prop $*$ Dimujer $+\beta_{3}$ prop $* D 30-44+\beta_{4}$ prop $*$ Dmas $44+\beta_{5}$ prop $* \exp +\varepsilon$ atvales $=\alpha+\beta_{1}$ prop $+\beta_{2}$ prop $*$ Dimujer $+\beta_{3}$ prop $* D 30-44+\beta_{4}$ prop $*$ Dmas $44+\beta_{5}$ prop $* \exp +\varepsilon$

- Promociones no congruentes no monetarias

atregalos $=\alpha+\beta_{1}$ prop $+\beta_{2}$ prop $*$ Dmujer $+\beta_{3}$ prop $* D 30-44+\beta_{4}$ prop $*$ Dmas $44+\beta_{5}$ prop $* \exp +\varepsilon$ atsorteos $=\alpha+\beta_{1}$ prop $+\beta_{2}$ prop $*$ Dmujer $+\beta_{3}$ prop $* D 30-44+\beta_{4}$ prop $*$ Dmas $44+\beta_{5}$ prop $* \exp +\varepsilon$

El parámetro $\beta_{1}$ permitirá conocer el efecto principal que tiene la propensión general hacia la promoción sobre el atractivo de los distintos tipos de incentivos promocionales. Por su parte, $\beta_{2}$ recogerá el efecto interacción entre la propensión a la promoción y el sexo que permitirá el contraste de la hipótesis H3. $\beta_{3}$ y $\beta_{4}$ medirán el efecto interacción entre la propensión a la promoción y la edad (H4). Finalmente, $\beta_{5}$ permitirá conocer el efecto interacción entre la propensión y la experiencia con el destino rural (H5).

El contraste de los parámetros se realizó a través del procedimiento de ajuste por mínimos cuadrados ordinarios utilizando el software estadístico Gretl. Tras la estimación de los parámetros se comprobaron las condiciones de validez del método de estimación. Los respectivos gráficos de probabilidad normal de los residuos mostraron que no había excesivas desviaciones en los puntos de la diagonal, no apreciándose por tanto problemas graves de falta de normalidad. Por lo que respecta a la multicolinealidad entre las variables explicativas los respectivos valores del Factor de Inflación de 
Varianza (FIV) y de Tolerancia permiten rechazar tal condición (véase tabla 5). Por último, para comprobar la presencia de heterocedasticidad se llevó a cabo el test de White (White, 1980) que puso de manifiesto que para las variables dependientes atractivo de los descuentos en precio (atdescuentos) y atractivo de cesión de un porcentaje del importe contratado para cuidado del medioambiente (atcuidado) existía tal problema. En ambos casos se utilizó el procedimiento de estimación bajo condiciones de heterocedasticidad HC3 propuesto por MACKINNON Y White $(1985)^{3}$ al ser recomendable para tamaño muestrales inferiores a 250 .

TABLA 5

\section{Pruebas de multicolinealidad entre las variables explicativas}

\begin{tabular}{|l|c|c|}
\hline Variables explicativas & FIV & Tolerancia \\
\hline prop & 1,56 & 0,64 \\
\hline prop*Dmujer & 1,19 & 0,84 \\
\hline prop*D30-44 & 1,62 & 0,62 \\
\hline prop*Dmas44 & 1,41 & 0,71 \\
\hline prop*exp & 1,10 & 0,91 \\
\hline
\end{tabular}

En la tabla 6 se muestran los resultados de las regresiones realizadas para cada una de las seis variables dependientes.

\footnotetext{
${ }^{3}$ Es ampliamente conocido que el modelo de regresión por mínimos cuadrados ordinarios (OLS) asume la existencia de homocedasticidad en los errores entre las observaciones (igualdad de varianzas). Si tal asunción no se cumple, la estimación de los errores estándar puede estar sesgada y afectar a la significación de los parámetros. Por esta razón se han desarrollado distintos métodos de estimación consistentes bajo condiciones de heterocedasticidad (heteroscedasticity-consistent, $\mathrm{HC}$ ): $\mathrm{HC} 0, \mathrm{HC} 1, \mathrm{HC} 2$ y HC3. Todos estos métodos HC están basados en una aproximación de la matriz de varianzas-covarianzas de los coeficientes de regresión estimados usando el cuadrado de los residuos en una regresión OLS $\left(\mathrm{e}_{\mathrm{i}}^{2}\right.$, donde $\left.\mathrm{e}_{\mathrm{i}}=\mathrm{y}_{\mathrm{i}}-\mathrm{x}_{\mathrm{i}} \beta\right)$. Los cuatro métodos propuestos difieren en cómo se utiliza el cuadrado de los residuos en el método de estimación. HC0 es la corrección clásica propuesta por White (1980) que funciona bien en muestras amplias, pero no tan bien en muestras más pequeñas. MacKinnon y White (1985) propusieron tres mejoras respecto al método original (HC1, HC2 y HC3), de las cuales Long y Ervin (2000) obtuvieron que la aproximación HC3 es la mejor para el caso de muestras pequeñas (muestras inferiores a 250). Los programas estadísticos más populares tales como SPSS no implementan ninguno de estos métodos de corrección por heterocedasticidad, lo que nos llevó a utilizar el programa GRETL, un software econométrico sofisticado y de código abierto (http://gretl.sourceforge.net/gretl_espanol.html) ampliamente utilizado por economistas y referenciado en libros y artículos científicos.
} 
TABLA 6

\section{Resultados de la regresión}

\begin{tabular}{|c|c|c|c|c|c|c|}
\hline & \multicolumn{2}{|c|}{$\begin{array}{c}\text { Promociones no congruentes } \\
\text { monetarias }\end{array}$} & \multicolumn{2}{|c|}{$\begin{array}{c}\text { Promociones no congruentes } \\
\text { no monetarias }\end{array}$} & \multicolumn{2}{|c|}{ Promociones congruentes } \\
\hline & $\begin{array}{l}\text { Atdescuentos } \\
\text { Coef. (valor p) }\end{array}$ & $\begin{array}{r}\text { Atvales } \\
\text { Coef. (valor } p)\end{array}$ & $\begin{array}{r}\text { Atregalos } \\
\text { Coef. (valor } p)\end{array}$ & $\begin{array}{r}\text { Atsorteos } \\
\text { Coef. (valor } p \text { ) }\end{array}$ & $\begin{array}{l}\text { Atexcursiones } \\
\text { Coef. (valor } p)\end{array}$ & $\begin{array}{r}\text { Atcuidado } \\
\text { Coef. (valor } p)\end{array}$ \\
\hline constante & $3,35992(<0,001$ & $\begin{array}{r}2,82085 \\
(<0,001 * * *)\end{array}$ & $\begin{array}{r}2,17202 \\
(<0,001 * * *)\end{array}$ & $\begin{array}{r}2,14968 \\
(<0,001 * * *)\end{array}$ & $\begin{array}{r}3,45469 \\
(<0,001 * * *)\end{array}$ & $\begin{array}{r}3,66285 \\
(<0,001 * * *)\end{array}$ \\
\hline $\operatorname{prop}\left(\beta_{1}\right)$ & $\begin{array}{r}0,07596 \\
(0,020 * *)\end{array}$ & $\begin{array}{r}0,07680 \\
\left(0,047^{* *}\right)\end{array}$ & $\begin{array}{r}0,13559 \\
(0,001 * * *)\end{array}$ & $\begin{array}{r}0,09617 \\
\left(0,018^{* *}\right)\end{array}$ & $\begin{array}{r}0,03870 \\
(0,307)\end{array}$ & $\begin{array}{r}0,04179 \\
(0,267)\end{array}$ \\
\hline $\begin{array}{l}\text { prop*Dmujer } \\
\left(\beta_{2}\right)\end{array}$ & $\begin{array}{r}0,03059 \\
(0,028 * *)\end{array}$ & $\begin{array}{l}0,03387 \\
\left(0,086^{*}\right)\end{array}$ & $\begin{array}{r}0,03144 \\
(0,133)\end{array}$ & $\begin{array}{r}0,03289 \\
(0,111)\end{array}$ & $\begin{array}{l}0,03490 \\
(0,072 *)\end{array}$ & $\begin{array}{r}0,01825 \\
(0,286)\end{array}$ \\
\hline $\begin{array}{l}\text { prop*D30-44 } \\
\left(\beta_{3}\right)\end{array}$ & $\begin{array}{r}-0,02259 \\
(0,151)\end{array}$ & $\begin{array}{r}-0,04370 \\
\left(0,060^{*}\right)\end{array}$ & $\begin{array}{r}-0,08505 \\
(<0,001 * * *)\end{array}$ & $\begin{array}{r}-0,06118 \\
(0,012 * *)\end{array}$ & $\begin{array}{l}-0,05313 \\
\left(0,020^{* *}\right)\end{array}$ & $\begin{array}{r}-0,01357 \\
(0,422)\end{array}$ \\
\hline $\begin{array}{l}\text { prop*Dmas44 } \\
\left(\beta_{4}\right)\end{array}$ & $\begin{array}{r}-0,00439 \\
(0,818)\end{array}$ & $\begin{array}{l}-0,05009 \\
\left(0,089^{*}\right)\end{array}$ & $\begin{array}{r}-0,04747 \\
(0,129)\end{array}$ & $\begin{array}{r}-0,04825 \\
(0,117)\end{array}$ & $\begin{array}{r}-0,01823 \\
(0,527)\end{array}$ & $\begin{array}{r}-0,03584 \\
(0,200)\end{array}$ \\
\hline $\operatorname{prop}^{*} \exp \left(\beta_{5}\right)$ & $\begin{array}{r}-0,00015 \\
(0,821)\end{array}$ & $\begin{array}{r}0,00130 \\
(0,191)\end{array}$ & $\begin{array}{r}-0,00092 \\
(0,383)\end{array}$ & $\begin{array}{r}0,00122 \\
(0,242)\end{array}$ & $\begin{array}{r}0,00078 \\
(0,426)\end{array}$ & $\begin{array}{r}0,00002 \\
(0,998)\end{array}$ \\
\hline & $\begin{array}{r}\mathrm{R}^{2}: 0,107 \\
\mathrm{R}^{2} \text { corregido: } 0,079 \\
\mathrm{~F}(5,158): 3,718 \\
\text { Valor } \mathrm{p}: \\
0,003289 * * *\end{array}$ & $\begin{array}{r}\mathrm{R}^{2}: 0,094 \\
\mathrm{R}^{2} \text { corregido: } \\
0,065 \\
\mathrm{~F}(5,158): 3,264 \\
\text { Valor } \mathrm{p}: \\
0,007828 * * *\end{array}$ & $\begin{array}{r}\mathrm{R}^{2}: 0,131 \\
\mathrm{R}^{2} \text { corregido: } \\
0,104 \\
\mathrm{~F}(5,158): 4,777 \\
\text { Valor p: } \\
0,000429 * * *\end{array}$ & $\begin{array}{r}\mathrm{R}^{2}: 0,106 \\
\mathrm{R}^{2} \text { corregido: } \\
0,077 \\
\mathrm{~F}(5,158): 3,733 \\
\text { Valor } \mathrm{p}: \\
0,003197 * * *\end{array}$ & $\begin{array}{r}\mathrm{R}^{2}: 0,069 \\
\mathrm{R}^{2} \text { corregido: } \\
0,040 \\
\mathrm{~F}(5,158): 2,361 \\
\text { Valor } \mathrm{p}: \\
0,042462^{*}\end{array}$ & $\begin{array}{r}\mathrm{R}^{2}: 0,033 \\
\mathrm{R}^{2} \text { corregido: } \\
0,002 \\
\mathrm{~F}(5,158): 1,277 \\
\text { Valor } \mathrm{p}: \\
0,276285\end{array}$ \\
\hline
\end{tabular}

$* * * \mathrm{p}<0,01 * * * \mathrm{p}<0,05 * \mathrm{p}<0,1$.

En todos los casos, a excepción de la variable dependiente atcuidado, se observa un efecto global significativo de las variables explicativas sobre el atractivo promocional dado que el test $\mathrm{F}$ resulta significativo. No obstante, el valor explicativo global del modelo es bajo dado que los R-cuadrado no superan en ningún caso 0,14. Esto en principio resulta bastante lógico dado que para contrastar las hipótesis propuestas se han planteado modelos de regresión que tratan de explicar una medida de actitud hacia las promociones utilizando únicamente la propensión hacia las mismas y varios efectos interacción de ésta con el sexo, la edad y la experiencia del turista con el destino.
Previo al contraste de hipótesis, los resultados mostraron que la propensión a la promoción ejerce un efecto positivo y significativo sobre el atractivo de las promociones de ventas con incentivos no congruentes con el turismo rural, ya sean monetarios (descuentos y vales) o no monetarios (regalos y concursos) $(p<0,05)$. En cambio el parámetro $\beta_{1}$ para los incentivos promocionales congruentes con la actividad de turismo rural (excursiones y cuidado del medioambiente) no resultó significativo $(p>0,1)$ en ningún caso.

La hipótesis H3 plantea que la influencia de la propensión a las promociones en el atractivo de las distintas herramientas promocionales en 
turismo rural será mayor para las mujeres que para los hombres, tanto en el caso de las promociones congruentes ( $\mathrm{H} 3 \mathrm{a})$ como en las no congruentes monetarias ( $\mathrm{H} 3 \mathrm{~b}$ ) y no congruentes no monetarias $(\mathrm{H} 3 \mathrm{c})$. El parámetro $\beta_{2}$ que recoge el efecto interacción entre la propensión y el hecho de ser mujer resultó ser significativo $(\mathrm{p}<0,1)$ y positivo sólo para las promociones basadas en descuentos y vales (incentivos no congruentes monetarios) y en excursiones y actividades al aire libre (incentivo congruente). Para el caso de los incentivos promocionales basados en regalos, descuentos y cuidado del medioambiente, a pesar de que el parámetro tiene un valor positivo en la línea propuesta por la hipótesis planteada, no resultó significativo $(p>0,1)$. Estos hallazgos permiten confirmar parcialmente $\mathrm{H} 3$ a y totalmente $\mathrm{H} 3 \mathrm{~b}$, no permitiendo confirmar $\mathrm{H} 3 \mathrm{c}$.

Según la hipótesis H4 cuanto más joven sea el cliente del alojamiento rural mayor será la influencia de la propensión a las promociones en el atractivo de las distintas herramientas promocionales utilizadas. Dado que la edad se ha transformado en dos variables dummy (D30-44, Dmas44) tomando como categoría de referencia el tramo de edad más joven de 18 a 29 años, el contraste de la hipótesis requiere revisar el valor y la significación de los parámetros $\beta_{3} \mathrm{y}$ $\beta_{4}$. El análisis de datos muestra que en todos los casos ambos parámetros presentan valores negativos lo cual indica que el efecto de la propensión sobre el atractivo promocional en las categorías de mayor edad es menor respecto a la categoría de referencia (18-29 años). No obstante, $\beta_{3}$ no resulta significativo $(\mathrm{p}>0,1)$ para los descuentos en precio y para el cuidado del medioambiente y $\beta_{4}$ sólo resulta significativo $(p<0,1)$ para los vales de noches extra en destino. Estos hallazgos sólo permiten confirmar parcialmente la hipótesis H4. Es decir, dicha afirmación se cumple completamente para los vales de descuento al resultar significativos $\beta_{3}$ y $\beta_{4}$, y parcialmente para los sorteos, regalos y las excursiones. No se cumple en ningún caso ni para los incentivos basados en descuentos ni para aquellos basados en el cuidado del medioambiente.

Finalmente en la hipótesis H5 se establecía que a mayor experiencia con el destino rural mayor influencia de la propensión a las promociones en el atractivo promocional. En este caso los hallazgos permiten rechazar totalmente dicha hipótesis ya que no se ha encontrado un efecto interacción significativo $(\mathrm{p}>0,1)$ entre la propensión y la experiencia con el destino en ninguno de los casos.

\section{CONCLUSIONES E IMPLICACIONES}

Los resultados obtenidos en esta investigación tienen importantes implicaciones no solo para la literatura sobre comportamiento del consumidor sino también para la literatura en turismo en lo referente al proceso de decisión de una estancia vacacional en un alojamiento rural. Además, las conclusiones que se derivan de este trabajo resultan especialmente útiles para los profesionales que gestionan destinos turísticos rurales, que podrán lograr incrementar la atracción de nuevos clientes, haciendo más atractivos sus productos turísticos a través de un uso adecuado de los incentivos promocionales.

Respecto a las implicaciones académicas cabe señalar, en primer lugar, la aportación al conocimiento de los factores que condicionan la eficacia de las promociones de ventas. Aunque es un tema ya tratado en la literatura de marketing, sin embargo, no lo ha sido tanto en el campo del turismo, en general, y menos aún en el del turismo rural, en particular. Como se ha puesto de manifiesto en la literatura quedan muchos interrogantes por resolver.

En este trabajo se ha demostrado que la eficacia de la promoción de ventas, medida en términos de atractivo, está condicionada por el tipo de incentivo promocional que se incluya. Concretamente se ha comprobado que las promociones de ventas congruentes (excursiones y cuidado del medio ambiente) con la actividad 
de turismo rural son más atractivas que las no congruentes no monetarias (regalos y sorteos). Estos resultados están en consonancia con la literatura dado que la actitud y por tanto la eficacia de la promoción va a ser significativamente mayor para las promociones congruentes con la actividad a desarrollar (TAYLOR Y CROCKER, 1981). Los turistas que realizan turismo rural están motivados por el contacto con el medio rural, buscan la tranquilidad y la relajación en la naturaleza, por lo que si se le ofrece un incentivo promocional acorde con las actividades que buscan desarrollar cuando realizan este tipo de turismo, el turista realizará un proceso de transferencia de actitud para evaluar la promoción, generando la misma actitud hacia el atractivo promocional que el que tiene hacia el turismo rural. Tal y como se pone de manifiesto en la teoría de la categorización (WANG et al., 2009).

Sin embargo, esto no se cumple para las promociones no congruentes monetarias basadas en descuentos en precio dado que son las que generan una mayor actitud en los consumidores. A pesar de la importancia de las promociones congruentes, las promociones que permitan a los turistas obtener un servicio a un precio más reducido las consideran más atractivas.

No obstante, en este trabajo se ha intentado comprobar si estos resultados se ven alterados en función del público objetivo al que se dirijan las promociones. Por lo que consideramos novedoso en el campo del turismo el análisis realizado del efecto conjunto de la propensión a la promoción, las variables demográficas (sexo y edad) y la experiencia del individuo con el destino turístico rural sobre el atractivo de seis tipos de incentivos promocionales distintos: promociones congruentes (excursiones y cuidado del medio ambiente), promociones no congruentes monetarias (descuentos en precio y vales para noches extra) y promociones no congruentes no monetarias (entrega de regalos y participación en concursos y sorteos).

El análisis realizado en este sentido ha dado lugar a unos hallazgos en la línea de los obteni- dos en otros trabajos previos (DUMAN Y MATTILA, 2003). Así, se confirma un efecto interacción significativo entre la propensión general a la promoción y el sexo sobre el atractivo de los incentivos promocionales no congruentes monetarios y un tipo de promoción congruente (excursiones). Mientras que este efecto no se ha mostrado para las promociones no congruentes no monetarias y el otro tipo de promoción congruente (cuidado del medio ambiente).

Respecto a la edad se ha hallado un efecto interacción significativo con la propensión en un tipo de promoción no congruente monetaria (vales descuento), parcialmente para las promociones no congruentes no monetarias y un tipo de promoción congruente (excursiones).

Finalmente, la experiencia con el destino turístico no parece moderar la influencia de la propensión a la promoción en el atractivo de ninguno de los incentivos promocionales considerados. En contra de lo que se pone de manifiesto en la literatura (RAGHUBIR Y CORFMAN, 1999; Duman y Mattila, 2003). Este hecho puede venir motivado por el tipo de turismo que estamos analizando, con unas características muy particulares. Se trata de individuos que buscan tranquilidad, contacto con la naturaleza e interacción con el medio ambiente.. Para estos turistas su elección del destino viene motivada principalmente por los recursos del destino y posteriormente por el atractivo del incentivo que le ofrecen. Los turistas rurales propensos a las promociones, ya sean monetarias o no monetarias y congruentes o no congruentes con este tipo de actividades turísticas las verán más o menos atractivas con independencia de que tengan mayor o menor experiencia con el destino de turismo rural. Para estos turistas no va a ser la experiencia lo que afecte al atractivo del incentivo promocional.

Respecto a las implicaciones profesionales cabe señalar que los resultados de esta investigación ayudarán a los responsables de la gestión de las empresas turísticas rurales a realizar campañas de promoción más eficaces. Necesi- 
dad clara para todo el sector turístico en general, dada la alta intensidad competitiva existente $\mathrm{y}$, especialmente para el turismo rural, si se considera además que se caracteriza por presentar empresas de pequeñas dimensiones con escaso presupuesto.

Este trabajo clarifica las actuaciones que deben llevar a cabo los gestores de alojamientos de turismo rural con respecto al tipo de incentivo promocional más atractivo para este tipo de turista. Si se desea aumentar la eficacia de las acciones promocionales y aumentar la actitud de los clientes hacia las mismas se aconseja apostar por las promociones congruentes y las promociones no congruentes monetarias basadas en descuentos en precios.

También se han obtenido resultados que permiten aumentar la efectividad de las promociones de ventas en función del público objetivo al que se dirijan. Los resultados permiten segmentar la población de turistas rurales pudiendo lanzar promociones más específicas en función al sexo y la edad de los individuos. En este sentido, cuando el público objetivo son mujeres, las promociones que generan una mejor actitud y son más eficaces son las promociones no congruentes monetarias y, dentro de las promociones congruentes, las basadas en el cuidado del entorno natural. La segmentación por edad permite conocer que si nos dirigimos a los más jóvenes podemos utilizar promociones congruentes y no congruentes ya sea monetarias como no monetarias. No obstante, la efectividad de la promoción, cuando la variable utilizada para segmentar el mercado es la edad, requiere seleccionar una herramienta específica de promoción para cada caso. La más idónea entre las promociones congruentes son las que se basan en excursiones y actividades al aire libre y entre las promociones no congruentes monetarias son los vales de descuento. Mientras que en el caso de promociones no congruentes no monetarias se puede utilizar tanto los concursos y sorteos como la entrega de regalos.

Por último, se ha comprobado que la experiencia con el destino rural no es una variable que han de contemplar los gestores en sus estrategias para conseguir la mayor efectividad posible de las promociones de ventas que ofrezcan a los turistas.

\section{LIMITACIONES Y FUTURAS LÍNEAS DE INVESTIGACIÓN}

Este trabajo no está exento de algunas limitaciones que es preciso señalar. La primera está relacionada con el tamaño muestral empleado (164). Con una muestra mayor los hallazgos serían más generalizables a toda la población de turistas rurales. Relacionado con esto, otra limitación tiene que ver con el área geográfica objeto de estudio, Andalucía. Sin lugar a dudas, los resultados serían más concluyentes si se hubiese tomado una muestra de turistas de otras zonas turísticas rurales en España situadas en entornos rurales bien diferentes, con una oferta de servicios distintos y con unos tipos de alojamientos diferentes a los existentes en esta Comunidad Autónoma. Además, cerca del $85 \%$ de los sujetos que componen la muestra proceden de la propia comunidad autónoma, de manera que sería necesario contrastar estos resultados con turistas rurales de otras zonas españolas con experiencias en turismo rural diferentes. En este sentido, una futura línea de investigación que se desprende es comprobar si los resultados obtenidos en este trabajo se corroboran utilizando muestras de turistas de otras zonas geográficas españolas, así como de otros países.

Otra futura línea de investigación futura tiene que ver con la realización de este análisis incluyendo otros incentivos promocionales congruentes y no congruentes (monetarios y no monetarios) distintos a los aquí seleccionados, así como otras cuestiones vinculadas con la personalidad de marca o el valor de marca.

Por último, también se propone como futura línea de investigación un análisis coste-beneficio de las diversas promociones para facilitar la toma de decisiones de las empresas de turismo rural. 


\section{REFERENCIAS BIBLIOGRÁFICAS}

Ailawadi, K.L., Neslin, S.A. y GedenK, K. (2001), "Pursuing the value-conscious consumer, store brands versus national brand promotions", Journal of Marketing, 65(1), 71-89.

ALFORD, B.L. Y Biswas, A. (2002), "The effects of discount level, price consciousness and sale proneness on consumer's price perception and behavioral intention", Journal of Business Research, 55(9), 775-783.

Álvarez, B. y VÁzQuez, R. (2005), "Efectividad de las promociones: Evidencias empíricas sobre la relación fidelidad versus categoría de producto", Revista de Economía y Empresa, 23(54/55), 11-28.

Álvarez, B. y VÁzquez, R. (2008), "Efecto de las promociones en precio sobre la selección por el consumidor entre marcas del fabricante y marcas del distribuidor", Revista Europea de Dirección y Economía de la Empresa, 17(2), 31-42.

Ávila, R. y BARrado, D.A. (2005), "Nuevas tendencias en el desarrollo de destinos turísticos, marcos conceptuales y operativos para su gestión y planificación", Cuadernos de Turismo, 15, 27-43.

BARKe, M. (2004), "Rural Tourism in Spain", International Journal of Tourism Research 6(3), 137-149.

BaWA, K. y Shoemaker, R.W. (1989), "Analyzing incremental sales from a direct mail coupon promotion", Journal of Marketing, 53(3), 66-78.

BlANCO F.J. (1996), “Fundamentos de la política comunitaria y española en materia de turismo rural". Estudios Turísticos, 131, 25-49.

BlatTBERG, R.C. y Neslin, S.A. (1990), "Sales promotion: Concepts, methods and strategies, en Papatla, P. y Krishnamurthi, L. (1996), "Measuring the dynamic effects of promotions on brand choice", Journal of Marketing Research, 33(1), 20-36.

CAMISÓN, C. (2000), "Strategic and information technologies in the hospitality business:
An empirical analysis", International Journal of Hospitality Management, 19(2), 125143.

CANTOR N. y Mischel, W. (1979), "Prototypes in person perception", Advances in Experimental Social Psychology, 12, 3-52.

CARPenter, J. y MoOre, M. (2007), "US consumers' perceptions of non-price retail promotions". International Journal of Retail \& Distribution Management, 36(2), 111-123.

Chandon, P., Wansink, B. Y Laurent, G. (2000), "A benefit congruency framework of sales promotion effectiveness", Journal of Marketing, 64(4), 64-81.

ChandRashekaran, R. y Grewal, D. (2003), "Assimilation of advertised reference prices: The moderating role of involvement", Journal of Retailing, 79(1), 53-62.

Consejería de Turismo, Comercio y DeporTES DE LA Junta de ANDAlucía, (2004), "Estudio sobre el turismo en Andalucía", disponible en: http://www.juntadeandalucia.es/turismocomercioydeportelabsys/abwe bp.exe/X6102/ID3846/, 15/06/2011.

D'Astous, A. Y JACOB, I. (2002), "Understanding consumer reactions to premium-based promotional offers", European Journal of Marketing, 36(11/12), 1270-1286.

D'Astous, A. y Landreville, V. (2003). “An experimental investigation of factors affecting consumers' perceptions of sales promotions", European Journal of Marketing, 37(11/12), 1745-1761.

Delvecchio, D. (2005), "Deal-prone consumers' response to promotion: The effects of relative and absolute promotion value", $P s y$ chology and Marketing, 22(5), 373-392.

Dowling, G.R. y UnCles, M. (1997), "Do Customer Loyalty Programs Really Work?", Sloan Management Review, 38(4), 71-82.

Duman, T. y Mattila, A.S. (2003), "A logistic regression analysis of discount reveiving behavior in the cruise industry, Implication for cruise marketers", International Journal of Hospitality \& Tourism Administration, 4(4), 45-57. 
EUROPEAN COMISSION (2010), "Europe 2020 strategy for sustainable growth and jobs", disponible en: http://ec.europa.eu/commission_2010-2014/president/news/statements/pdf/20100210_3_en.pdf, 11/07/2011.

Fleischer, A. y Felsenstein, D. (2000), “Support for rural tourism: Does it make a difference?", Annals of Tourism Research, 27(4), 1007-1024.

FodNess, D. Y MurRaY, B. (1997), "Tourist information search", Annals of Tourism Research. 24(3), pp. 503-523.

FUENTES, R. (1995), El turismo rural en España. Especial referencia al análisis de la demanda. Ministerio de Comercio y Turismo, Secretaría General de Turismo.

GARCíA, J.L. (1996), "El turismo rural como factor diversificador de rentas en la tradicional economía agraria", Revista de Estudios Turísticos, 132, 47-61.

Gázquez Abad, J.C. y De CANNière M.H. (2010), "Impacto promocional vs. relacional del mailing personalizado en el comportamiento de compra: El papel moderador de las características del consumidor" Revista Española de Investigación de Marketing ESIC, 14(1), 57-89.

Haley, R.I. y Baldinger, A.L. (1991), "The ARF copy research validity project", Journal of Advertising Research, 31(2), 11-32.

Henderson, C. (1987), Sales promotion segmentation: Refining the deal-proneness construct, Documento de trabajo, The Amos Tuck School of Business Administration, Dartmouth College, Hanover, Nh.

Instituto DE Estudios TuRísticos, IET (2008), Informe anual de Familitur. Ministerio de Industria, Turismo y Comercio. Madrid.

Instituto Nacional de Estadística, INE (2010), "Encuesta de ocupación de alojamientos turísticos", disponible en: www.ine.es, 11/07/2011.

Instituto NACiOnAL DE EstadísticA, INE (2011), "Encuesta de ocupación de alojamientos turísticos", disponible en: www.ine.es, 11/07/2011.
Judge, G.G., HILl, R.C., GRIFFITHS, W.E., LÜTKEPOHL, H. Y LEE, T.C. (1988), Introduction to the theory and practice of econometrics, John Wiley \& Sons: New York.

KRISHNA, A. Y ZHANG, Z.J. (1999), "Short or long duration coupons. The effect of the expiration date on the profitability of coupon promotions", Marketing Science, 45(8), 1044-1056.

Krishna, A., Currim, I.S. y SHOEMAKer, R.W. (1991), "Consumer perceptions of promotional activity", Journal of Marketing, 55(2), 416.

KwoK, S. y UnCles, M. (2002), Sales promotion effectiveness: The impact of culture at an ethnic-group level, Documento de Trabajo, Universidad de New South Wales, Sydney.

KwoK, S. y UnCles, M. (2005), "Sales promotion effectiveness: The impact of consumer differences at an ethinic-group level", Journal of Product \& Brand Management, 14(2/3), 170-186.

Kwon, K.N. Y KwON, Y.J. (2007), “Demographics in sales promotion proneness, A sociocultural approach", Advances in Consumer Research, 34, 288-294.

Laroche, M., Pons, F., Zgollia, N., CerveLLON, M.C. Y KIM, C. (2003), "A model of consumer response to two retail sales promotion techniques", Journal of Business Research, 56(7), 513-522.

LiChtenstein, D.R., BURTON, S. y NeteMEyeR, R.G. (1997b), "Psychological correlates of a processes to deals: A domain specific", Advances in Consumer Research, 24(1), 274-280.

Lichtenstein, D.R., Netemeyer, R.G. y BuRTON, S. (1990), "Distinguishing coupon proneness from value consciousness: An acquisition-transaction utility theory perspective", Journal of Marketing, 54(3), 54-67.

Lichtenstein, D.R., Netemeyer, R.G. Y BuRTON, S. (1995), "Assessing the domain specificity of deal proneness, A field study", Journal of Consumer Research, 22(3), 314326. 
Lichtenstein, D.R., Netemeyer, R.G. Y BuRTON, S. (1997a), "An examination of deal proneness across sales promotion type: A consumer segmentation perspective", Journal of Retailing, 73(2), 283-297.

LiChTENSTEIN, D.R., RidWAY, N.M. Y NETEMEYER, R.G. (1993), "Price perceptions and consumer shopping behabiour: a field study", Journal of Marketing Research, 30(2), 234-245.

LONG, J. S., Y ERVIN, L. (2000), "Using heteroscedasticity-consistent standard errors in the linear regression model," The American Statistician, 54, 217-224.

LuCAS, A.F. y BowEN, J.T. (2002), "Measuring the effectiveness of casino promotions", Hospitality Management, 21(2), 189-202.

LYNCH, J. Y SCHULER, D. (1994), “The matchup effect of spokesperson and product congruence. A schema theory interpretation", Psychology and Marketing, 11(5), 417-445.

MacKenzie, S.B., Lutz, R.J. y Belch, G.E. (1986), "The role of attitude toward the ad as a mediator of advertising effectiveness: a test of competing explanations", Journal of Marketing Research, 23(2), 130-143.

MacKinnon, J.G. Y White, H. (1985), "Some heteroskedasticity consistent covariance matrix estimators with improved finite sample properties", Journal of Econometrics, 29(3), 305-325.

Martínez, E. y Montaner, T. (2004), "Características de los consumidores propensos a las promociones", Investigaciones Europeas de Dirección y Economía de la Empresa, 10(3), 37-53.

Martínez, E. y Montaner, T. (2007), “Análisis del perfil psicográfico de los consumidores propensos a las promociones de productos de compra frecuente", Cuadernos de Economía y Dirección de la Empresa, 30, 63-92.

Martínez, E.; Montaner, T. y Pina, J.M. (2007), "Estrategia de promoción e imagen de marca: influencia del tipo de promoción, de la notoriedad de la marca y de la con- gruencia de beneficios", Revista Española de Investigación de Marketing ESIC, 11(1), 27-51.

MAZUNDAR, T. y PAPATLA, P. (1995), "Gender differences in prince and promotion response", Pricing Strategy \& Practice, 3(1), 2133.

Mela, C.F., Gupta, S. y Lehmann, D.R. (1997), "The long-term impact of promotion and advertising on consumer brand choice", Journal of Marketing Research, 34(2), 248261.

Mela, C.F., Jedidi, K. y Bowman, D. (1998), "The long-term impact of promotions on consumer stockpiling behaviour", Journal of Marketing Research, 35(2), 250-262.

MitTAL, B. (1994), "An integrated framework for relating diverse consumer characteristics to supermarket coupon redemption", Journal of Marketing Research, 31(4), 533-544.

Monty, B. y SKIDMORE, M. (2003), "Hedonic pricing and willingness to pay for bed and breakfast amenities in Southeast Wisconsin", Journal of Travel Research, 42(2), 195199.

Okumus, R. y Hemmington, N. (1998), "Barriers and resistance to change firms: An investigation at unit level", International Journal of Contemporary Hospitality Management, 10(7), 283-288.

Organización Mundial del Turismo, OMT (2007), "El turismo contribuirá a dar solución a los retos del cambio climático y la pobreza, retos que deben abordarse de forma holística", disponible en: http://www.unwto.org/newsroom/news/sp/press det.php?id $=623,16 / 06 / 2008$.

PALAZÓN, M. (2005), "La propensión a la promoción del consumidor: Una nueva perspectiva basada en beneficios", Revista Española de Investigación de Marketing ESIC, 9(2), 111-133.

Palazón, M. y Delgado, E. (2009), "La evaluación de las promociones monetarias y no monetarias según la propensión a la promoción del consumidor", Revista Española de 
Investigación de Marketing ESIC, 13(1), 3570.

Parreño Selva, J.; Leeflang P.S.H. y Van Doorn, J. (2009), "Efecto de las promociones del precio sobre la venta de productos no promocionados: papel moderador del tipo de visita del cliente" Revista Española de Investigación de Marketing ESIC, 13(2), 73-90.

Peattie, K. y Peattie, S. (1996), "Promotional competitions: A winning tool for tourism marketing", Tourism Management, 17(6), 433-442.

Pechtl, H. (2004), "Profiling intrinsic deal proneness for HILO and EDLP price promotion strategies", Journal of Retailing and Consumer Services, 11(4), 223-233.

Price, L.L., FeICK, L.F. y Guskey-Federouch, A. (1988), "Couponing behaviors of the market maven, Profile of a super couponer", Advances in Consumer Research, 15(1), 354-359.

RAGHuBir, P. y CORFMAN, K. (1999), "When do price promotions affect pretrial brand evaluations?", Journal of Marketing Research, 36 (2), 211-222.

Roehm, M.L., Pullins, E.B., y Roehm JR., H.A. (2002), "Designing loyalty-building programs for packaged goods brands", Journal of Marketing Research, 39(2), 202-213.

Satorra, A. y Bentler, P.M. (1986), "Some robustness properties of goodness of fit statistics in covariance structure analysis", American Statistical Association, Proceedings of the Business and Economic Statistics Section, 549-554.

SCHNEIDER, L.G. Y CURRIM, I.S. (1991), “Consumer purchase behaviors associated with active and passive deal-proneness", International Journal of Research in Marketing, 8(3), 205-222.

SHIMP, T.A. Y KaVAS, A. (1984), "The theory of reasoned action applied to coupon usage", Journal of Consumer Research, 11(3), 795809.

SiVAKUMAR, K. y RAJ, S.P. (1997), "Quality tier competition, How price change influences brand choice and category choice", Journal of Marketing, 61(3), 71-84.

Swaminathan, S. y Bawa, K. (2005), "Category-specific coupon proneness: The impact of individual characteristics and categoryspecific variables", Journal of Retailing, 81(3), 205-214.

TAYLOR, S.E. Y CROCKER, J. (1981), "Schematic bases of social information processing", en E. Tory Higgins, C.P. HeRman y M.P. ZanNA, Social Cognition. The Ontario Symposium on Personality and Social Psychology, Hillsdale, NJ. Erlbaum, 89-134.

Teunter, L., Wierenga, B. Y Kloek, T. (1999), "Sales promotion effects and the deal prone consumer: Analisys of consumer reactions through a magnifying-glass", 28 Emac Conference, Berlin, 1-15.

Thornton, P.R., Shaw, G. Y Williams, A.M. (1997), "Tourist group holiday decisionmaking and behaviour: the influence of children", Tourism Management, 18(5), 287-297.

VALDÉs, L. (1996), "El turismo rural en España". En PEDREÑo, A. (Director) y MONFORT, V. (Coordinador), Introducción a la Economía del Turismo en España. Civitas, Madrid.

VAN RAAIJ, W. y FranCKEN, D. (1984), "Vacation decisions, activities, and satisfactions", Annals of Tourism Research, 11(1), 101-112.

VICDAN, H. y SUN, J. (2008), "Towards a framework for understanding compulsive buyers online: Exploring the effects of online sales promotions", 2008 Swdsi Meeting.

Villalba Merlo, F.J. (2005), "La promoción de ventas y los beneficios percibidos por el consumidor". Revista Europea de Dirección y Economía de la Empresa, 14(3), 209-222.

WAKEFIELD, K.L. Y BARNES, J.H. (1996), "Retailing hedonic consumption, a model of sales promotion of a leisure service", Journal of Retailing, 72(4), 409-427.

WALTERS, R.G. (1991), "Assessing the impact of retail price promotions on product substitution, complementary purchase, and interstore sales displacement", Journal of Marketing, 55(2), 17-28. 
Wang, S., Beatty, S.E. Y Mothersbaugh, D.L. (2009), "Congruity's role in website attitude formation", Journal of Business Research, 62(6), 579-672.

White, H. (1980). "A heteroskedastic-consistent covariance matrix estimator and a direct test of heteroskedasticity". Econometrica, 48, 817-838.

Yoon, H.J., Nusair, K., Parsa, H.G., y NaIPAUL, S. (2010), "Price formats, discounts, and consumers' perceptions: A comparison between hospitality and non-hospitality industries", Journal of Foodservice Business Research, 13(1), 51-65.

Zalatan, A. (1998), "Wives' involvement in tourism decision processes". Annals of Tourism Research, 25(4), 890-903.
Fecha recepción: 17/07/2011

Fecha aceptación: 29/11/2011 